\title{
Eike-Christian Hornig
}

\section{»Camera obscura« - Der italienische Senat und seine Mitglieder auf Lebenszeit: Vom Abstellgleis zur Nebenregierung ${ }^{1}$}

Das italienische Parlament gilt im internationalen Vergleich als eine Besonderheit, da es mit dem Abgeordnetenhaus und dem Senat über zwei gleichberechtigte und direkt gewählte Kammern verfügt. Dieser »bicameralismo perfetto« ist seit den Umbrüchen der 1990er Jahre verstärkt in das Fadenkreuz institutioneller Reformen gerückt, da er als Quelle der Instabilität der Regierungen schnell auszumachen war. Da aber eine Kopplung des Senats an eine Dezentralisierung bzw. Föderalisierung Italiens bislang ausblieb, ist der Senat nach wie vor der »machtvolle Zwillingsbruder der Abgeordnetenkammer «². Seit der Parlamentswahl im April 2006 steht er besonders im Mittelpunkt der Aufmerksamkeit, da die Regierung Prodi hier nur über eine hauchdünne Mehrheit verfügt. Bei einer symbolträchtigen Abstimmung über die Außenpolitik im Februar 2007 kam diese Mehrheit nicht zu Stande und stürzte die Regierung in die Krise. Dafür ausschlaggebend waren, neben der Abwesenheit zweier kommunistischer Senatoren der Regierungskoalition, auch zwei Senatoren auf Lebenszeit (SaL). Insbesondere der Pate der italienischen Politik, der mehrfache Ministerpräsident Giulio Andreotti sorgte mit einem kurzfristigen Stimmungswechsel für Kritik an der Rolle der Senatoren auf Lebenszeit als Zünglein an der Waage $^{3}$. Diese war zuvor schon im Dezember 2006 laut geworden, da der Haushalt der Regierung nur Dank der massiven Unterstützung der Senatoren auf Lebenszeit verabschiedet werden konnte, was Oppositionspolitiker wie der Vorsitzende der Oppositionspartei »Alleanza Nazionale«, Gianfranco Fini als undemokratisch kritisierten $^{4}$.

1 Für ihre Hinweise danke ich Ralf Kleinfeld, Anna Lena Etzbach und David Grewe.

2 Jörg Seisselberg, »Der italienische Senat: Machtvoller Zwillingsbruder der Abgeordnetenkammer«, in: Gisela Riescher / Sabine Ruß / Christoph Haas (Hg.), Zweite Kammern, München 2000, S. 202-219.

3 Gianna Fregonara, »Da Cossiga a Pininfarina, è giallo sul voto dei senatori a vita «, in: www.corriere. it, 28. Februar 2007.

4 Gianfranco Fini sagte dazu: »Wir kritisieren nicht das Recht der Senatoren abzustimmen, sondern die Tatsache, dass ohne diese Stimmen die Regierung keine Mehrheit hätte und die Abstimmung nicht Ausdruck des Wählermandates ist. Wir bewegen uns hier also außerhalb der demokratischen Logik, wenn man an der Regierung steht ohne über die Mehrheit derjenigen zu verfügen, die gewählt worden sind « (Ubersetzung Autor). Ohne Autor, "Manovra, Fini: Voto senatori a vita è contro la logica democratica«, www.repubblica.it, 17. Dezember 2006. 
Der vorliegende Aufsatz nimmt diese Ereignisse zum Anlass die Institution der Senatoren auf Lebenszeit und ihre Rolle im italienischen Parlament zu untersuchen. Er versteht sich dabei in einem größeren Zusammenhang als ein Beitrag zur Analyse der 2. Republik in Italien, deren Profil durch das Ineinandergreifen von Kontinuität und Wandel immer noch undeutlich ist. Während die Institution der Senatoren auf Lebenszeit formal unberührt geblieben ist, strahlt der Wandel in der Struktur des Parteienwettbewerbes und damit auch des parlamentarischen Wettstreites auf ihre Bedeutung und Charakterisierung aus. Denn durch die Wandlungsdynamiken des Parteiensystems sind die Senatoren auf Lebenszeit von der Peripherie in das Zentrum der Aufmerksamkeit und Bedeutung des parlamentarischen Prozesses im Senat gerückt.

Zunächst wird gezeigt, dass die SaL mit ihrer Kombination aus Nominierung und vollständigen parlamentarischen Kompetenzen im internationalen Vergleich eine Besonderheit sind, die auf wackeligen demokratietheoretischen Füßen steht. Der Aufsatz macht zweitens deutlich, dass der größte Teil der ernannten SaL ehemalige Spitzenpolitiker waren, so dass diese Institution zumindest in der 1. Republik von 1948 bis 1993 einen starken parteipolitischen Charakter hatte. Das war aber so lange unproblematisch, wie die dominierende Herrschaftslogik des »trasformismo « mit breiten informellen Mehrheiten, den Senatoren auf Lebenszeit in der parlamentarischen Praxis kein Gewicht zukommen ließ. Dies änderte sich mit dem Übergang zur 2. Republik 1994. Angesichts der rivalisierenden Parteienkoalitionen kommt den SaL bei knappen Mehrheiten tatsächlich die Rolle des Züngleins an der Waage zu. Zugleich droht bei der Fortschreibung der Ernennungstradition der 1. Republik eine Vereinnahmung durch den tagespolitischen Wettbewerb, da die Parteien bei Stimmenknappheit auf ihre SaL zurückgreifen werden. Insgesamt verträgt sich die Institution der Senatoren auf Lebenszeit schlechter mit der parlamentarischen Logik und Praxis der 2. Republik, als mit jener der 1. Republik. Schließlich analysiert der Aufsatz anhand von 100 Abstimmungen zwischen November 2006 und Februar 2007 die konkrete Rolle der SaL im Verhältnis zur Regierung Prodi, um erstmals ein genaues Bild über den inhaltlichen Stand der Senatoren auf Lebenszeit zu bekommen.

\section{Bestellmodus und Repräsentationsleistung des Italienischen Senates im internationalen Vergleich}

Hinsichtlich der demokratietheoretischen Bedeutung Zweiter Kammern ${ }^{5}$ stehen der Bestellmodus und ihre Repräsentationsleistung im Vordergrund, die wiederum

5 Degener weist hinsichtlich der Nummerierung der Kammern daraufhin, dass sich diese im Laufe der Zeit verkehrt hat, da z.B. in Dänemark, Schweden oder den Niederlanden die Erste Kammer die indirekt gewählte Kammer war. »Der Namenswechsel ist in anderen Ländern vermutlich auf den Legitimationsvorsprung der Direktwahl zurückzuführen, der es mit dem Siegeszug der parlamentarischen Demokratie nicht mehr opportun erschienen ließ, die volksnähere Kammer hinter die ständisch oder aristokratisch privilegierte zurücktreten zu lassen«. Ursula Degener, »Die Abschaffung des Zweikammerreichstags in Schweden ", in: Gisela Riescher / Sabine Ruß / Christoph Haas (Hg.), Zweite Kammern, München 2000, S. 353-367. 
mit dem vertikalen Staatsaufbau zusammenhängt. Christoph M. Haas unterscheidet vier Methoden der Abgeordnetenbestellung: Direkte Wahl, indirekte Wahl, Nominierung und ständestaatliche Vertretung. Zusätzlich ergibt sich noch mit der kombinierten Wahl in Belgien und Spanien eine Sonderform als fünfte ${ }^{6}$. Beim italienischen Senat kommen drei davon zusammen: Die direkte Wahl, die automatische Ernennung der ehemaligen Staatspräsidenten zu Senatoren auf Lebenszeit durch die Verfassung und die Nominierung von maximal fünf Bürgern durch den amtierenden Staatspräsidenten zu Senatoren auf Lebenszeit. Genau genommen handelt es sich in Italien, wie auch z.B. in Irland ${ }^{7}$ also um gemischte Bestellmodi, die aber anhand des dominanten Verfahrens charakterisiert werden. Neben Italien verfügen die Schweiz, Polen, Tschechien, Kroatien und Rumänien ebenfalls über eine direkt gewählte Zweite Kammer. Indirekt gewählt wird dagegen in Frankreich, den Niederlanden, Österreich und Deutschland ${ }^{8}$. Eine breite Nominierung gibt es in Europa nicht, während das britische House of Lords eine ständestaatliche Vertretung ist. Dass einzelne, einige oder alle Mitglieder einer Zweiten Kammer nicht unmittelbar demokratisch bestellt sind, ist im international Vergleich also nichts Ungewöhnliches.

Charakteristisch für Italien ist zweitens, dass es sich um einen unitarischen Staat mit einem Zweikammersystem handelt. Während alle großen, föderalen oder heterogenen Demokratien über zwei Kammern verfügen, gibt es unitarische Staaten mit und ohne Zweite Kammer. Daher besteht im internationalen Vergleich auch ein eher ausgeglichenes Verhältnis beider Formen. Der Anteil der Zweikammersysteme wird von Salvatore Vasallo in seinem Vergleich von 46 Demokratien auf $57 \%$ beziffert ${ }^{9}$. Während in föderalen Systemen die Zweiten Kammern dem Prinzip des Mehrebenensystems oder der Heterogenität des Landes Rechnung tragen und somit ihre Legitimation in der Repräsentation subnationaler Ebenen (Deutschland, Schweiz)

6 Christoph M. Haas, "Sein oder nicht sein: Bikameralismus und die Funktion Zweiter Kammern «, in: Gisela Riescher / Sabine Ruß / Christoph Haas (Hg.), Zweite Kammern, München 2000, S. 2-17, hier S. 13.

7 Hier sitzen Vertreter zweier Universitäten, der Landwirtschaft, von Industrie und Handel und aus der Verwaltung. Außerdem werden von den 60 irischen Senatoren elf direkt vom Premierminister ernannt.

8 Wobei der Deutsche Bundesrat keine Zweite Kammer im eigentlichen Sinne darstellt, da er ein eigenständiges Verfassungsorgan ist. »Auch wenn der Bundesrat nicht als Zweite Kammer des deutschen Parlamentes konzipiert ist, nimmt er bei der Gesetzgebung eine Aufgabe war, die für das Zusammenspiel von zwei Kammern eines Parlamentes typisch ist.« Roland Sturm / Petra Zimmermann-Steinhart, Föderalismus. Eine Einfübrung, Baden-Baden 2005, S. 48.

9 Zur Vergleichsgruppe gehören folgende Ländern, die Salvatore Vasallo z.T. mit Abstrichen als Demokratien einordnet: USA, Kanada, Australien, Neuseeland, die 27 Mitglieder der EU, Norwegen, Schweiz, Island, Brasilien, Argentinien, Chile, Mexiko, Russland, Südafrika, Costa Rica, Kolumbien, Venezuela, Israel, Indien und Japan. Salvatore Vassallo, »Come le seconde camere rappresentano i 'territori'. Le elezioni dell'analisi comparata ", in: Stefano Ceccanti / Salvatore Vassallo (Hg.), Come chiudere la transizione. Cambiamento, apprendimento e adattamento nel sistema politico italiano, Bologna 2002, S. 339-358, hier S. 342. 
oder gesellschaftlicher Teilgruppen (Belgien) liegt, fehlt bei Zweiten Kammern in unitarischen Systemen diese Legitimitätsbasis.

Daher sieht Sabine Ruß diese in einem Legitimationsdilemma gefangen ${ }^{10}$, was schließlich zur Frage von Romy Messerschmidt führt: Wozu eigentlich Zweite Kammern in Zentralstaaten ${ }^{11}$ Je geringer der Unterschied zur Ersten Kammer ist, desto größer der Legitimationsdruck. Dennoch haben im Vergleich unitarischer Länder immerhin noch vier von zwölf Zweite Kammern eine de facto kongruente Repräsentationsleistung wie die jeweilige Erste. Italien gehört neben Rumänien, Japan und den Niederlanden in diese Gruppe. Bei ihnen unterscheidet sich zwar mit direkten und indirekten Wahlen die Bestellung, dennoch entscheidet die Proportionalität der Bevölkerung über die Besetzung. Die anderen acht unitarischen Staaten weisen einen anders definierten Repräsentationsschlüssel auf, z.B. das ständestaatliche Modell in Irland, Slowenien und Großbritannien oder die Repräsentation der Distrikte im ansonsten unitarischen Chile ${ }^{12}$. Riescher/Ruß halten allerdings fest, dass oft die Repräsentationsbasis Zweiter Kammern nicht eindeutig zu bestimmen ist. »In die Analyse der Repräsentationsfunktion einzubinden sind deshalb aktuelle Vertretungen und Vertretungszuschreibungen, die die Zweiten Kammern unabhängig von ihrer manifesten Funktionszuschreibung (...) tatsächlich wahrnehmen. Außer parteipolitischen Färbungen sind damit auch längerfristig überdauernde Strukturen zu beachten wie etwa die Sozialstruktur der Zweiten Kammern «13.

Zugleich gibt es auch Beispiele für die Abschaffung von Zweiten Kammern in Einheitsstaaten. Den Anfang in Europa machte Dänemark im Jahr 1953, nachdem in Neuseeland der »legislative council« im Jahr 1950 bereits abgeschafft worden war. In Dänemark besiegelte eine Volksabstimmung das Ende des »Landsting«. Dieser sollte sich bis dahin insbesondere durch Expertise und reflektierte Sachkenntnis auszeichnen. Dabei waren die beiden Kammern seit 1915 nahezu gleichberechtigt. Charakteristisch für die indirekt gewählten Mitglieder war, dass sie wesentlich älter waren, als jene der Ersten Kammer »Folketing «. So galt der »Landsting « als ein Altmännerparlament (»gammelmandsting «), wie Ursula Degener hervorhebt ${ }^{14}$. Wahrgenommen wurde die Zweite Kammer in Dänemark als ein Organ der Oberschicht, das aufgrund der Ähnlichkeit mit der Ersten Kammer zunehmend an Bedeutung

10 Sabine Ruß, »Die andere Kammer. Zur Repräsentationsleitung des Senats in der Fünften Republik «, in: Joachim Schild / Henrik Uterwedde (Hg.), Frankreichs V. Republik. Ein Regierungssystem im Wandel, Wiesbaden 2005, S. 105-126, hier S. 107.

11 Romy Messerschmidt, »Wozu Zweite Kammern in Zentralstaaten - das Beispiel des französischen Senats «, in: Werner J. Patzelt (Hg.), Parlamente und ibre Macht. Kategorien und Fallbeispiele institutioneller Analyse, Baden-Baden 2005, S. 47-87.

12 Salvatore Vasallo, "Come le seconde camere rappresentano i 'territori'. Le elezioni dell'analisi comparata«, aaO. (FN 9), S. 356.

13 Gisela Riescher / Sabine Ruß, "Zur Funktion von Zweiten Kammern in modernen Demokratien«, in: Gisela Riescher / Sabine Ruß / Christoph Haas (Hg.), Zweite Kammern, München 2000, S. 382-401, hier S. 388.

14 Ursula Degener, »Der dänische Landsting - nur vom historischen Interesse? «, in: Gisela Riescher / Sabine Ruß / Christoph Haas (Hg.), Zweite Kammern, München 2000, S. 337-352. 
verlor und schließlich abgeschafft wurde. Ein ähnliches Schicksal erfährt auch 1970 die Zweite (ehemals Erste) Kammer in Schweden (»Första Kammaren«). Ursprünglich als ein Oberhaus mit Adligen, Gutsbesitzern und hohen Beamten eingerichtet, unterschieden sich die Repräsentationsleistung der beiden Kammern dennoch geringfügig, da die Zweite nach einem indirekten lokalen Schlüssel besetzt war. Grundsätzlich wurde ihr dabei eine ähnliche Legitimation wie im dänischen Fall zugewiesen. Hier »saßen die - im Durchschnitt älteren - sachkundigen Politiker, die Experten; das Niveau war akademischer... « ${ }^{15}$. Abgeschafft wurde sie schließlich in einem Konsens der großen Parteien, da ihr die Förderung der Parteienzersplitterung und des (lokalen) Partikularismus zugeschrieben wurde.

Ganz ähnlich wie die abgeschafften Zweiten Kammern in Schweden und Dänemark soll sich der italienische Senat durch seine Eigenschaft als »Kammer der Erfahrung ${ }^{{ }^{16}}$ von der Ersten Kammer abheben, denn die Intention des Verfassungsgebers war es, den Senat zu einem Sammelpunkt politischer Kompetenz zu machen. Während der Abgeordnetenkammer, u.a. durch die Anwesenheit der Parteivorsitzenden, der Großteil der Aufmerksamkeit zukommt, gilt der Senat als der Versammlungsort der erfahrenen Politiker. Inwiefern das gelungen und ob das eine eigene Repräsentationsleitung ist, bleibt so zweifelhaft, wie die Methode, dieses zu bemessen. Zwar wird der Senat auch als die Kammer der Professoren bezeichnet, da diese eine der größten Berufsgruppe stell( $(t) e^{17}$. Dass der Senat deshalb eine klügere Gesetzgebung gewährleistet, bezeichnet Seißelberg aber zu Recht als blauäugig, auch wenn der Senat im Vergleich zur Kammer disziplinierter und produktiver arbeitet ${ }^{18}$. Außerdem ergibt sich kaum ein Unterschied beim Blick auf die Abgeordneten mit abgeschlossenem Hochschulstudium. Im Jahr 2001 waren es 72,2\% im Senat und $71,1 \%$ in der Kammer ${ }^{19}$. So bleibt nur, die Rolle der Kammer der Erfahrung auf das Alter der Senatoren zu stützen. Das lag mit einem Durchschnitt zwischen 53,6 (1994) und 55 Jahren (2001) tatsächlich etwa fünf Jahre über dem der Kammer und stieg 2006 auf 57,5 Jahre im Durchschnitt an. Die Senatoren auf Lebenszeit unterstreichen mit ihrem Durchschnittsalter von 87,2 Jahren (2007) vorzüglich die Repräsentationsidee ihrer Kammer.

Eine dritte Variationsmöglichkeit in Zweikammersystemen stellt die Kompetenzverteilung zwischen den Häusern dar. Hinsichtlich der Symmetrie oder Asymmetrie der Kompetenzen beider Kammern benennt Vasallo vier Grade der Asymmetrie und eine fünfte Stufe, auf der beide Kammern gleichberechtigt sind. In der ersten Stufe ist die Zweite Kammer der Ersten ganz klar untergeordnet und kann nur sym-

15 Ursula Degener, »Die Abschaffung des Zweikammerreichstags in Schweden «, in: Gisela Riescher / Sabine Ruß / Christoph Haas (Hg.), Zweite Kammern, München 2000, S. 357.

16 Gisela Riescher / Sabine Ruß, »Zur Funktion von Zweiten Kammern«, aaO. (FN 13), S. 389.

17 Gianni Piazza / Orazio Lanza, »Il ricambio dei parlamentari«, in: Gianfranco Pasquino (Hg.), Dall'Ulivo al governo Berlusconi, Bologna 2002, S. 239-274, hier S. 264.

18 Jörg Seißelberg, »Der italienische Senat«, aaO. (FN 2), S. 215

19 Gianni Piazza / Orazio Lanza, »Il ricambio dei parlamentari«, aaO. (FN 17), S. 264. 
bolisch auf den Gesetzgebungsprozess Einfluss nehmen. Die zweite Stufe kennzeichnet die Möglichkeit der Zweiten Kammer Gesetzesvorlagen zurückzuschicken und eigene Vorschläge zu machen. Auf der dritten Stufe besteht die Möglichkeit begrenzter eigener Rechte und eines effektiven Vetos. Die vierte Stufe kennzeichnen größtenteils gleiche Rechte mit einigen Einschränkungen. Auf der fünften Stufe ist die Symmetrie erreicht ${ }^{20}$. Es gibt im Vergleich der 27 Zweikammersysteme nur fünf bei denen eine symmetrische Kompetenzenverteilung besteht: Mexiko, USA, Chile sowie wiederum Italien und Rumänien. Die anderen 22 Fälle verteilen sich auf die verschiedenen Asymmetriestufen. Außerdem ist die Kombination aus identischer Kompetenzenverteilung und identischer Repräsentationsleistung selten (Italien/Rumänien), da im internationalen Vergleich Zweite Kammern in der Mehrheit den Ersten Kammern nachgeordnet und gleichzeitig nach einem eigenen Repräsentationsschlüssel besetzt sind.

Der Italienische Senat verfügt also über die gleiche Legitimation und Repräsentationsleitung wie die Abgeordnetenkammer, was eine parteipolitische Dopplung produziert. Auch wenn sich der Wahlschlüssel mit der Einteilung nach Regionen vom Einheitswahlkreis der Abgeordnetenkammer formal leicht unterscheidet, ist das Ergebnis nahezu analog. Verstärkt durch die Symmetrie mit der Ersten Kammer, ist das von Ruß benannte Legitimationsdilemma in Italien (und Rumänien) am intensivsten. Die Zweiten Kammern in Dänemark und Schweden, waren im Vergleich zum italienischen Senat noch unterschiedlicher - und wurden trotzdem abgeschafft. Eine ins 19. Jahrhundert zurück reichende Tradition hatten diese auch.

Wenn die Berechtigung des Senates schon auf den dünnen Beinen der Erfahrung seiner (älteren) Mitglieder beruht, stellt sich hinsichtlich der Senatoren auf Lebenszeit noch mehr die Legitimationsfrage. Seißelberg führt dazu eine historische Erklärung an, die auf den Italienischen Einigungsprozess unter dem monarchischen "Statuto albertino « und auf die Gründung der Republik im Geiste des Widerstandes nach dem 2. Weltkrieg zurückgeht. Im Königreich Piemont, unter dessen Führung die Einigung Italiens vollzogen wurde, gab es eine Zweite Kammer, deren Abgeordnete durch den König nominiert wurden. Diese Tradition stand während der Verhandlungen in der Verfassungsversammlung (»Assemblea Costituente«) zwischen 1946 und 1948 zur Disposition, da Kommunisten und Sozialisten ein Einkammerund Christdemokraten und Liberale ein Zweikammersystem bevorzugten. Als Kompromiss wurde die Zweite Kammer als Spiegelbild der Ersten eingerichtet. Für dieses Entgegenkommen, stimmten die Linken auch der Beibehaltung der Nominierung von Senatsmitgliedern durch das Staatsoberhaupt zu, wenn auch nur in einem kleinen Rahmen ${ }^{21}$.

Praktisch verfügen die SaL aber nur über eine schwache demokratische Legitimation, da diese auf einer Kette basiert, an deren Anfang die Abgeordneten der beiden Kammern stehen, die den Staatspräsidenten wählen und als einzige über eine direkte

20 Salvatore Vasallo, »Come le seconde camere rappresentano i >territori««, aaO. (FN 9), S. 358.

21 Jörg Seißelberg, »Der italienische Senat«, aaO. (FN 2), S. 203. 
Legitimation verfügen. Dass der Staatspräsident nach dem Ausscheiden in den Senat einzieht, ist Teil seiner Berufung und der ersten indirekten Legitimationsstufe. Die Ernennung der anderen Senatoren auf Lebenszeit verlängert die Legitimationskette um ein weiteres Glied. Diese schwache demokratische Legitimation der italienischen Senatoren auf Lebenszeit sticht aber nur im direkten Vergleich mit ihren Senatskollegen, nicht aber im internationalen Vergleich hervor. Denn die beiden Bestellmodi der SaL erscheinen angesichts der Pluralität der Verfahren anderer Zweiter Kammern, nicht als ungewöhnlich. Was hervorsticht ist, dass die Senatoren auf Lebenszeit, anders als analog bestellte Parlamentarier, über volle parlamentarische Kompetenzen verfügen. Es handelt sich also um die einmalige und demokratietheoretisch ungünstige Kombination von schwacher demokratischer Legitimation und vollen parlamentarischen Kompetenzen.

\section{Die Senatoren auf Lebenszeit: Nobelpreisträger und Parteipolitiker}

Insgesamt hat es 42 Senatoren auf Lebenszeit gegeben, von denen zehn als ehemalige Staatspräsidenten automatisch in diese Funktion gerückt sind. Während zwischen 1963 und 1983 im Durchschnitt etwa sieben SaL dem Senat angehörten, nahm ihr Anteil ab 1983 deutlich zu. In der Phase der politischen Umbrüche zu Beginn der 1990er Jahre wird mit zehn Senatoren auf Lebenszeit $(3,17 \%)$ der durchschnittliche Höchstwert erreicht. Fraglich ist allerdings, ob es hier einen Zusammenhang gibt $^{22}$. Bedeutende Veränderungen im Parteiensystem manifestierten sich dann erst mit der Parlamentswahl 1992 und mit den Korruptionsskandalen (Tangentopoli) im Jahr 1993. Von da an hält sich die Anzahl etwa zwischen neun und zehn, sinkt aber nach 2000 auf das Niveau der 1960er bis 1980er Jahre ab. Die Schwankungen hängen dabei von der Ernennungspraxis des jeweiligen Staatpräsidenten, von der Lebensdauer der Senatoren und von der Periodik des Präsidentenamtes ab.

22 Zum einen waren zuvor mit dem ehemaligen Staatspräsident Sandro Pertini im Februar 1990 und Cesare Merzagora (der seit 1963 im Amt war) im Mai 1991 zwei SaL gestorben. Daraufhin ernannte Staatspräsident Francesco Cossiga im Mai und Juni 1991 fünf neue SaL (u.a. Giulio Andreotti und Giovanni Agnelli). Ein Jahr später schied Cossiga selbst aus dem Amt und rückte somit in den Senat ein, so dass sich die Zahl der SaL seit 1990 um 3 Mandate auf 10 erhöhte. Gegen einen Zusammenhang mit der politischen Krise spricht zweitens, dass das Referendum über die Veränderung des Wahlrechtes von 1991, das wesentlich zur Öffnung der Blockade der Parteienherrschaft beigetragen hat, erst eine Woche später stattfand. Anna Capretti, Die Öffnung der Machtstrukturen durch Referenden in Italien, Frankfurt/M. 2001, S. 139-148. 


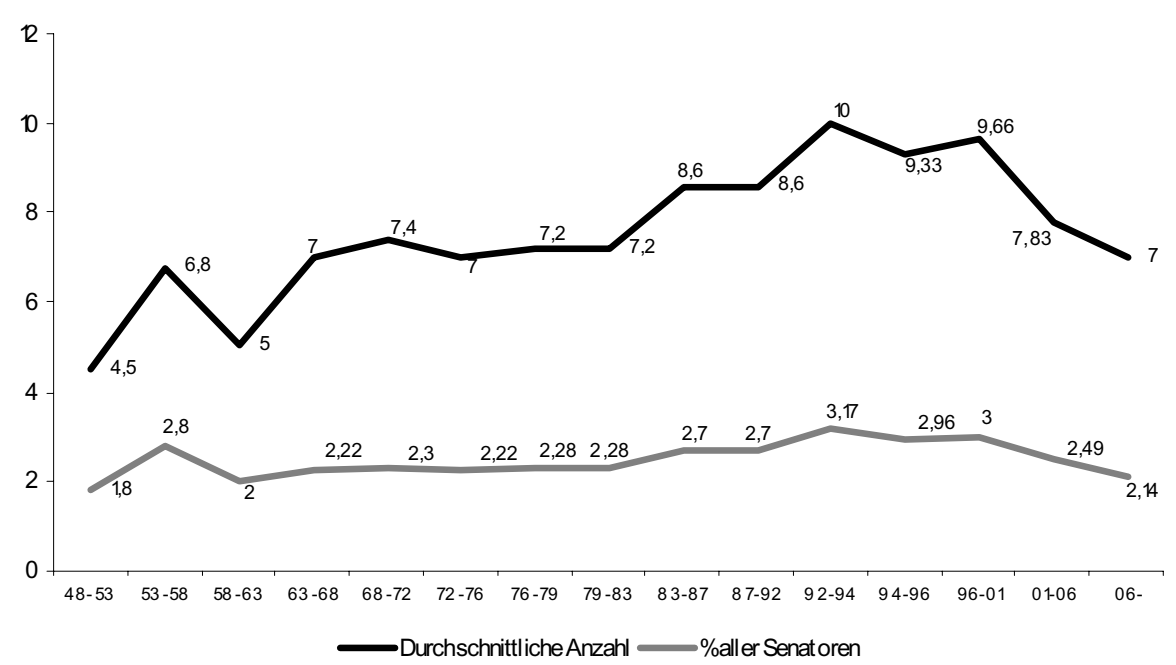

Abbildung 1.: Anzabl der Senatoren auf Lebenszeit und ibr prozentualer Anteil an der Gesamtmenge aller Senatoren im Durchschnitt pro Legislaturperiode (Quelle: Eigene Darstellung).

Die Gruppe der ernannten Senatoren auf Lebenszeit setzt sich insbesondere aus ehemaligen langjährigen Spitzenpolitikern zusammen. Von den insgesamt 32 Ernennungen machen sie mit 14 etwa $43,7 \%$ aus. Hierzu gehören allein vier ehemalige Präsidenten des Senates (Cesare Merzagora, Bartolomeo Ruini, Amintore Fanfani, Giovanni Spadolini) und zwei Präsidenten der Abgeordnetenkammer (Giovanni Leone und Giorgio Napolitano). Des weiteren haben neun der 32 ernannten SaL ein oder mehrere Male ein Ministeramt bekleidet; sechs von ihnen waren sogar mal Regierungschef (Ferruccio Parri, Giovanni Leone, Amintore Fanfani, Giovanni Spadolini und Giulio Andreotti), bzw. Vize-Regierungschef (Pietro Nenni). Hier bestätigt sich die Aussage von Tsebelis, dass es im Italien der 1. Republik eine unheimlich stabile personelle Kontinuität gegeben hat ${ }^{23}$. Dabei endet eine politische Spitzenkarriere nach vielen Stationen oftmals mit dem Amt des Senators auf Lebenszeit.

Mit Giovanni Leone, Amintore Fanfani und Giovanni Spadolini haben drei Schlüsselfiguren der 1. Republik diesen Karrierelauf verfolgt. Hinzufügen ließen sich noch Don Luigi Sturzo als Mitbegründer der Christdemokratischen Partei (DC) und Giulio Andreotti, der als mehrfacher Minister und Ministerpräsident ebenfalls die Präsidentschaft des Senates nach der Wahl 2006 anstrebte, aber nicht erlangte. Giovanni Leone und Giorgio Napoletano konnten diese Karriere sogar noch verlängern, da sie als Senatoren auf Lebenszeit zu Staatspräsidenten wurden, auch wenn Napoletano nie Regierungschef war. Emilio Colombo gehörte als Minister mehrfach der Italienischen Regierung an und wurde 1977 zum Präsidenten des Europaparlamentes gewählt.

23 George Tsebelis, Veto players. How political Institutions work, New York 2002, S. 5. 
Von den anderen 19 Senatoren auf Lebenszeit stellen sieben Künstler die größte Gruppe. Zu ihnen gehört u.a. der Literaturnobelpreisträger Eugenio Montale, aber auch der weltberühmte Dirigent Arturo Toscanini. Des weiteren wurden sechs Wissenschaftler zum SaL ernannt. Zu ihnen gehört der Philosoph Norberto Bobbio, die Medizin-Nobelpreisträgerin Rita Levi Montalcini oder der Archäologe Umberto Zanotti Bianco, der auch lange Jahre Präsident des Italienischen Roten Kreuzes war. Neben ihnen fungierten drei Unternehmer als SaL, von denen zwei eng mit dem größten Konzern des Landes verbunden waren. Vittorio Valletta war Präsident von FIAT, während Giovanni Agnelli mit seiner Familie hinter dem Konzern stand. Der Designer Sergio Pininfarina war auch Präsident des Italienischen Industrieverbandes »Confidustria«. Schließlich sind noch der Journalist und Befreiungskämpfer Leo Valiani und der Jurist Francesco De Martino zu erwähnen. De Martino hat sich dabei durch den einmaligen Vorgang hervorgehoben, dass er ein Jahr vor seinem Tod 2001 als Senator auf Lebenszeit offiziell der Fraktion der Linksdemokraten (»Democratici di Sinistra - ULIVO«) beitrat und damit seine parteipolitische Neutralität aufgab.

Ohne Zweifel gehörten die genannten Politiker zu den prominentesten und etabliertesten der 1. Republik. Das Amt des Senators auf Lebenszeit stand also zumindest zur Phase der 1. Republik insbesondere langjährigen Spitzenpolitikern zu. Der externe Zufluss durch Senatoren auf Lebenszeit, die keine ehemaligen Spitzenpolitiker sind, überwiegt zwar knapp, doch behält die Institution einen sehr stark parteipolitisch gefärbten Charakter. Inwiefern diese Ernennungstradition sich in der 2. Republik fortsetzt, ist dabei noch nicht abzusehen, da es seit 1994 bislang nur wenige Ernennungen gegeben hat. Diese standen nicht im Zeichen der Parteipolitik. Im Jahr 2001 wurde die Medizin-Nobelpreistägerin Rita Levi-Montalcini, im Januar 2003 der Unternehmer Emilio Colombo, und im September 2005 der Unternehmer Sergio Pininfarina ernannt.

\section{Die Senatoren auf Lebenszeit zwischen "trasformismo" und "catch-all-Koalitionen"}

Die Institution der Senatoren auf Lebenszeit ist durch den Wandel von der 1. zur 2. Republik formal nicht berührt worden. Trotz ihrer insgesamt geringen quantitativen Bedeutung, bestand seit 1948 häufig die Möglichkeit für die SaL als Zünglein an der Waage zu funktionieren, da es bei 16 von insgesamt 60 Regierungen (26,6\%) formal sehr knappe Mehrheiten in der Zweiten Kammer gab. In diesen 16 Fällen umfasste die Unterstützung der Regierung im Senat nur zwischen 47,7 und 52\% der Senatoren. Somit waren die Senatoren auf Lebenszeit mit ihren zwischen 2 und 3\% theoretisch prädestiniert um als Zünglein an der Waage in Frage zukommen. Dabei konzentrierten sich elf ${ }^{24}$ dieser 16 Fälle auf die zweite und dritte Legislaturperiode

24 Hiervon können allerdings zwei Regierungen (De Gasperi VIII und Fanfani I) abgezogen werden, da sie nicht zu Stande kamen. Übrig bleiben: Leone I (IV. Legisl. 1963); Leone II, Rumor II, Andreotti I (V. Legisl. 1968); Moro IV und V (VI. Legisl. 1972); Andreotti III, IV, V (VII. Legisl. 1976), Cossiga I (VIII. Legisl. 1979); Fanfani VI (IX. Legisl. 1983). 
zwischen 1953 und 1963. In weiteren 19 von 60 Fällen lag die Mehrheit der jeweiligen Regierung im Senat über der komfortablen 55\%-Hürde, so dass die SaL keine Rolle spielten. Das gilt auch für die elf Minderheitsregierungen, da die Stimmen der SaL alleine nicht zur Mehrheit reichten.

Außerdem ergeben sich vier weitere Sonderfälle. Die ersten drei Regierungen der ersten Legislaturperiode verfügten im Senat nicht über eine Mehrheit an gewählten Vertretern, da die neue Verfassung eine Übergangsregelung vorsah, die die Zusammensetzung des Senats betraf (»III. disposizione transitoria«). Neben den 243 gewählten Senatoren wurden weitere 106 ernannt. Bei diesen handelte es sich um ehemalige Parlamentsangehörige der vor-faschistischen Zeit, um Mitglieder der Verfassungsgebenden Versammlung und Verfolgte des faschistischen Regimes. Mit Beginn der zweiten Legislaturperiode schieden diese wieder aus und nur noch die SaL blieben ${ }^{25}$. Zweitens handelte es sich in der zwölften Legislaturperiode um die Regierung von Lamberto Dini, der als so genannter unabhängiger »tecnico « eine überparteiliche Regierung mit dem Vertrauen des Staatspräsidenten führte ${ }^{26}$.

Dabei zeigt die Analyse, dass die Regierungen in der 1. Republik sich in der Praxis fast immer auf die Zusammenarbeit mit den Oppositionsparteien stützen konnten. Mit Ausnahme vielleicht der zweiten Regierung Amintore Fanfani, ergab sich kaum Spielraum für das Votum der Senatoren auf Lebenszeit ${ }^{27}$. Während sich scheinbar immer wieder Variationen in der Breite der externen Unterstützungen ergaben, dominierte kontinuierlich eine bestimmte Herrschaftstechnik die Mehrheits-

25 http://senato2003.senato.it/funz/cost/transito.htm\#III.

26 Piero Calandra, I governi della Repubblica. Vicende, Formule, Regole, Bologna 1996, S. 522-532.

27 (1) Die Regierung Pella I, die zweite der 2. Legislaturperiode, war eine Minderheitsregierung, die nur 141 Tagen bestand hatte. Ministerpräsident Pella legte den Schwerpunkte auf eine »administrative "Führung und weniger auf die Durchsetzung des DC Programms, womit er die Tolerierung durch die Republikaner (PRI), Liberalen (PLI) und Monarchisten gewann. Außerdem standen die Sozialdemokratien (PSDI) und die Postfaschisten (MSI) neutral zur Regierung. (2) In der Regierung Scelba fand sich die Koalition aus DC, PDSI und PLI offiziell zusammen, aber nur mit 49,3\% der Senatoren. Allerdings wurde die Regierung durch die Monarchisten und Republikaner inoffiziell unterstützt, so dass die Mehrheit de facto größer war. (3) Dies galt auch für die folgende Regierung Segni I, die fünfte der 2. Legislaturperiode. (4) Die anschließende DC-Minderheitsregierung von Ministerpräsident Adone Zoli konnte sich keiner offiziellen Tolerierung erfreuen, sondern musste sich ein Jahr lang wechselnde Mehrheiten suchen. (5) Die Parlamentswahl 1958 produzierte eine DC-PSDI-Regierung, die gelegentlich von den Sozialisten (PSI) unterstützt wurde. Das Wahlergebnis von Ministerpräsident Amintore Fanfani fiel mit drei Stimmen Mehrheit in der Kammer und sieben Stimmen im Senat (52\%) allerdings äußerst knapp aus. Nach mehreren Abstimmungsniederlagen in der Abgeordnetenkammer, trat die Regierung nach 209 Tagen zurück. Es folgten ihr drei Minderheitsregierungen der DC: (6) Antonio Segni II, (7) Fernando Tamborini und (8) Amintore Fanfani III, die jeweils über 49\% der Senatoren verfügten und durch die Liberalen, Monarchisten und Postfaschisten unterstützt wurden. (9) Die vierte Regierung Fanfani, die sich 449 Tage im Amt halten konnte, stützte sich in einer Koalition aus DC, PSDI und PRI immerhin auf 52\% der Senatoren. Ebd. S. 103, 152, 577. 
findung: der so genannte »trasformismo « als »institutionalisierte Form der Elitenkooperation ${ }^{28}$. Charakteristisch dafür war die Einbeziehung oppositioneller Kräfte zur Sicherung der eigenen Mehrheit bzw. der situative Wechsel zwischen der Regierungs- zur Oppositionsbank, wie Stefan Köppl schreibt ${ }^{29}$. »Gerade die Fachkommissionen des Parlamentes wurden zu strategischen Spielfeldern, um den für die Mehrheitsbildung notwendigen Grundkonsens zu produzieren. Dieser Kanal wurde häufig zum Beschluss von Gesetzen benutzt, welche die Oppositionsparteien in politisch minder relevanten Angelegenheiten begünstigten, um dafür im Ausgleich, nach dem Prinzip des log-rolling, die Zustimmung der Opposition zu Gesetzesvorlagen der Regierung im Plenum zu erhalten « ${ }^{30}$. Während die Grenzen zwischen Regierung und Opposition zunehmend verschwammen, wurden auf Kompromissen basierende Gesetze mit großen Mehrheiten verabschiedet. »Selbst als die Christdemokraten in der Phase zwischen 1948 und 1953 die parlamentarische Mehrheit besaßen, wurden 70\% der Gesetzesvorlagen mit Zustimmung der Oppositionsparteien, darunter der Kommunistischen Partei, verabschiedet. In späteren Jahrzehnten stieg dieser Prozentsatz der einheitlich gebilligten Gesetzesvorlagen sogar auf $90 \% \ll^{31}$. Das betrifft auch einen weiteren Fall aus dem Jahr 1972, als die zweite Regierung Andreotti formal nur über 47,8\% der Senatoren verfügte.

Die Instabilität der Regierungen war zwar evident, doch mehr oberflächlich, während die personelle und inhaltliche Kontinuität sehr groß war. Die tatsächliche Konstruktion der Koalition oder das Ausmaß der bekundeten Tolerierung einer Minderheitsregierung wird angesichts des »trasformismo « als Fassade erkennbar. Seinen Höhepunkt fand der »trasformismo " im so genannten historischen Kompromiss (»compromesso storico«), als die Kommunistische Partei (PCI) zwischen 1976 und 1979 die Minderheitsregierungen der DC massiv unterstützte ${ }^{32}$. Es wird also deutlich, dass die Existenz der SaL in der Phase der 1. Republik angesichts des »trasformismo « bedeutungslos bzw. nur von symbolischem Charakter war.

Die letzten beiden Regierungen der 1. Republik von Giuliano Amato (Juni 1992 bis April 1993) und Carlo Azeglio Ciampi (April 1993 bis April 1994) verfügten ebenfalls nur über eine sehr schmale formelle Mehrheit von $51,7 \%$ im Senat. Wie die erste Regierung Berlusconi ab 1994, sind beide als Sonderfälle vor dem Hintergrund der Veränderungen im politischen System zu Beginn der 1990er Jahre, insbesondere der Parlamentswahl 1992, zu betrachten. Die Wähler straften die etablierten Parteien $1992 \mathrm{ab}$ und signalisierten einen tief greifenden Wunsch nach Veränderung. Ma-

28 Elisabeth Fix, Italiens Parteiensystem im Wandel. Von der Ersten zur Zweiten Republik, Frankfurt/M. 1999, S. 97.

29 Stefan Köppl, Das politische System Italiens, Wiesbaden 2007, S. 63.

30 Elisabeth Fix, Italiens Parteiensystem im Wandel, aaO. (FN 28), S. 109.

31 Ebenda.

32 Elisabeth Fix sieht den Hintergrund für dieses gemeinsame Vorgehen der Kommunisten und Christdemokraten in den ökonomischen und sozialen Krisen der 1970er Jahre, die einen temporären Schulterschluss der beiden großen Parteien notwendig machten. Elisabeth Fix, Italiens Parteiensystem im Wandel, aaO. (FN 28), S. 113. 
rio Segni bezeichnet die Wahlen 1992 als die letzten der »democrazia bloccata «33. Auch Sani und Segatti sprechen von den Wahlen 1992 als den letzten der 1. Repub$\operatorname{lik}^{34}$. Die Wahlen hatten primär einen Anti-System-Charakter, der sich in der massiven Abstrafung sowohl der Regierungsparteien, als auch der anderen etablierten Parteien äußerte. Der Schaden für das bisherige Parteiensystem wurde dabei nicht zuletzt durch die Wahlerfolge der Lega Nord deutlich. Von herben Wahlverlusten, dem politischen Funktionsverlust und den Korruptionsaffären schwer getroffen und in Lethargie erstarrt, kam es zu einer immer stärkeren Aufspaltung der DC, deren Rest sich im Januar 1994 in Partito Populare italiano (PPI) umbenannte.

Angesichts der massiven Wahlverluste der bisherigen Staatstragenden Parteien, der zunehmenden Stärke der populistischen Protestpartei Lega Nord (8,6\% der Stimmen) und der Schwäche der ehemaligen Kommunisten, erwies sich die Mehrheitsbildung als äußerst schwierig. Ehemalige Bestandteile der Regierung standen nicht mehr zur Verfügung (wie die Republikaner), während die Lega Nord dieses (noch) kategorisch ablehnte. Der ebenfalls neue Staatspräsident Oscar Luigi Scalfaro plädierte für ein Übergangskabinett, das mit Parteipolitikern und unabhängigen Experten besetzt sein sollte. Die daraufhin unter Ministerpräsident Giuliano Amato eingesetzte Regierung symbolisierte eine große Koalition aller bedeutenden Lager der 1. Republik. Offiziell wurde die Regierung von der traditionellen »quadripartito (DC, PSDI, PSI und PLI) getragen. Calandra charakterisiert diese als eine offene Regierung, die sich zugleich aber um die Unterstützung der ehemaligen Kommunisten (umbenannt in PDS), der Republikaner (PRI) und auch der Grünen bemühte. Insbesondere den Christdemokraten war klar, dass in dieser Phase des Umbruchs auch die ehemaligen Kommunisten berücksichtigt werden mussten. Dementsprechend bildeten sich zu verschiedenen Themen auch variable Mehrheiten $^{35}$. Zugleich wurde der Empfehlung des Staatspräsidenten Rechung getragen und auch Fachexperten in hohe Regierungsämter berufen. In dieser Art von informeller großer Koalition waren die Senatoren auf Lebenszeit nicht von Bedeutung.

Die Überlegungen zur Nachfolgeregierung waren wesentlich durch die Referenden vom 18. April 1993 geprägt. Deren Wirkung beschreibt Capretti wie folgt: »Neben der symbolträchtigen Streichung der staatlichen Parteienfinanzierung war das wichtigste Ergebnis dieser Abstimmung die Einführung eines Mehrheitswahlrechts für den Senat, die von 82,7\% der Wähler klar befürwortet wurde. Das Ergebnis war ein deutliches Signal, den Einfluss der Parteien zu reduzieren und die große personelle Kontinuität durch neue, unverbrauchte Politiker zu beenden « ${ }^{36}$. Staatspräsi-

33 Mario Segni, »Un nuovo referendum per completare la 'rivoluzione interrotta' «, in: Pierluigi Mantini (Hg.), Verso il bipolarismo in Italia. Referendum elettorale, riforme costituzionali e partiti politici, Mailand 1998, S. 139-146, hier S. 139.

34 Giacomo Sani / Paolo Segatti, »Fratture sociali, orientamenti politici e voto: Ieri e oggi«, in: Roberto D'Alimonte / Stefano Bartolini (Hg.), Maggioritario finalmente? La transizione elettorale 1994 - 2001, Bologna 2002, S. 249-281, hier S. 249.

35 Piero Calandra, I governi della Repubblica, aaO. (FN 26), S. 475.

36 Anna Capretti, Die Öffnung der Machtstrukturen durch Referenden in Italien, aaO. (FN 22), S. 152. 
dent Scalfaro entschied eine Woche nach den Volksabstimmungen, den damaligen Leiter der Staatsbank Carlo Azeglio Ciampi mit der Führung einer Regierung zu betrauen, da dieser ein finanzpolitischer Fachmann war (was in Zeiten der damaligen ökonomischen Krise sehr wichtig war) und gleichzeitig eine große Distanz zu den politischen Parteien symbolisierte. Die Aufgabe seiner Regierung war es, die Reform des politischen Systems, vor allen Dingen des Wahlsystems, im Sinne der Volksabstimmungen voran zu treiben. So wurde diese Regierung auch als »governo del referendum ${ }^{37}$ bezeichnet. Ihre Stützen waren der Staatspräsident und die moralische Verpflichtung der Parteien zur Mitarbeit. Die Senatoren auf Lebenszeit spielten in dieser heißen Phase des Übergangs keine Rolle.

Im Zusammenhang mit der ersten Regierung von Silvio Berlusconi 1994 kam es zu einem ersten spektakulären Fall, bei dem die Senatoren auf Lebenszeit bei einer wichtigen Abstimmung im Senat den Ausschlag gaben. Die Wahl von Berlusconi zum Regierungschef wäre ohne die SaL gescheitert. Für Berlusconi stimmten 159 Senatoren, während 153 gegen ihn votierten. Das Quorum lag bei 158 Stimmen. Die Stimmen von drei Senatoren auf Lebenszeit verhalfen Berlusconi also ins $\mathrm{Amt}^{38}$.

Der Wandel von der 1. zur 2. Republik ist für die Senatoren auf Lebenszeit also von wesentlicher Bedeutung, da sich die Funktionslogiken des parlamentarischen Abstimmungsprozesses veränderten und sie in einem veränderten Umfeld auch eine veränderte Wirkung erzielten. Zwar gab es in der 1. Republik trotz der informellen Mehrheiten auch oft Abstimmungsniederlagen der Regierung, wie Köppl festhält. Demnach fiel die Erfolgsrate von Regierungsvorlagen von ca. 90\% in den 1950ern bis auf 50\% in den 1980er Jahren und 30\% in der Umbruchphase in den 1990ern. Dennoch wurden sehr viele Gesetze verabschiedet, die nach der Logik des »trasformismo « zu Stande gekommen sind. Köppl attestiert dabei mit dem zweiten Kabinett Berlusconi eine erhebliche gestiegen Durchsetzungskraft der Regierung ${ }^{39}$. Der »historische Kompromiss « zwischen Christdemokraten und Kommunisten ist das beste Beispiel dieser informellen Koalition gewesen, wenn auch schon ein wenig zu formell.

In Zeiten rivalisierender Parteikoalitionen in der 2. Republik ist kein Platz mehr für den »trasformismo«. Das, durch das neue Mehrheitswahlrecht, tendenziell eher bipolar ausgeprägte Parteiensystem mit rivalisierenden Parteienkoalitionen machte es der Regierung Berlusconi schwer die Vorgehensweise des »trasformismo « effektiv anzuwenden. Dabei hatte es Berlusconi vielleicht sogar noch am einfachsten, da sich noch ein dritter Parteienpol in der Mitte hatte durchsetzen können, dessen Senatoren Berlusconi (nach seiner Wahl mit Hilfe der SaL) extern unterstützten ${ }^{40}$. Neben den beiden Lagern Mitte-Links und Mitte-Rechts gab es das »centro«, das sich aus den »Populari« (PPI) und dem »Patto Segni« zusammensetzte. Sie verfügten in der Kammer über 7,3\% und im Senat über 9,8\% der Abgeordneten, die fast aus-

37 Piero Calandra, I governi della Repubblica, aaO. (FN 26), S. 497.

38 Ohne Autor: "Ciampi: si chiami fase 2 o 1 ora il governo si gioca tutto «, in: Corriere della Sera, 17. Dezember 1994, S. 11.

39 Stefan Köppl, Das politische System Italiens, aaO. (FN 29), S. 163.

40 Piero Calandra, I governi della Repubblica, aaO. (FN 26), S. 514. 
schließlich auf die PPI zurückgingen. Während die folgenden Regierungen der 2. Republik über verhältnismäßig stabile Mehrheiten im Senat verfügten, verschwindet der dritte Parteienpol in der Mitte bis 2001 vollständig. Demnach hat sich sowohl die Anzahl der politischen Lager (»schieramenti«), als auch die Anzahl der Parteienkoalitionen zwischen der ersten Wahl der 2. Republik 1994 und 2001 reduziert. Während die einfache Anzahl an Lagern 1994 drei und effektive Anzahl 2,5 betrug, liegen 2001 beide Werte bei 2. Die Anzahl der effektiven Koalitionen im Senat sank von 3,7 1994 auf 2,1 im Jahr 2001. ${ }^{41}$ Trotz der augenscheinlichen Bipolarität konnte sich »das Mehrheitswahlrechts als Gründungsmythos der 2. Republik « ${ }^{42}$ aber nicht durchsetzen und alle gewünschten Effekte produzieren. So kennzeichnen das Parteiensystem nicht catch-all-Parteien sondern catch-all-Koalitionen ${ }^{43}$. Diese stellen in einem bipolaren Parteienwettbewerb abwechselnd die Regierungen, die sich trotz breiterer Koalitionen, als erstaunlich stabil erwiesen haben. Aldo di Virgilio hat dies als Proportionalisierung des Mehrheitswahlrechts bezeichnet ${ }^{44}$. Der Mischcharakter des Systems verstärkte sich noch durch das neue Verhältniswahlrecht der Regierung Berlusconi 2005, da es z.B. für die Kammer eine Mehrheitsprämie vorsah.

\section{Die Krise der Regierung Prodi im Februar 2007 und die Senatoren auf Lebenszeit}

Die im internationalen Vergleich sehr hohe Anzahl an verabschiedeten Gesetzen steht einer breiteren empirischen Auswertung des Abstimmungsverhaltens der Senatoren auf Lebenszeit im Wege. Seit der Gründung der Republik 1948 bis zum Ende der 13. Legislaturperiode hat das italienische Parlament circa 14800 Gesetze produziert, während der Deutsche Bundestag in genauso vielen Jahren nur 5993 Gesetze beschloss. Der Durchschnitt liegt damit in Italien bei ungefähr 278 Gesetzen pro Jahr. Köppl weist allerdings daraufhin, dass mit dem Übergang zur 2. Republik auch eine verminderte Gesetzesproduktion feststellbar würde. Insgesamt ist die hohe Anzahl an Gesetzen durch das Ausschusssystem zu erklären ${ }^{45}$. Daher soll an dieser Stelle anhand von 100 Abstimmungen im Senat beispielhaft ihr Verhältnis zur aktuellen Regierung Prodi untersucht werden ${ }^{46}$. Diese ist das jüngste Beispiel für

41 Stefano Bartolini / Alessando Chiaramonte / Roberto D'Alimonte, »Maggioritario finalmente? «, in: Roberto D’Alimonte / Stefano Bartolini (Hg.), Maggioritario finalmente? La transizione elettorale 1994 - 2001, Bologna 2002, S. 363-380, hier S. 374

42 Mauro Calice, La terza repubblica. Partiti contro Presidenti, Rom 2006, S. 11.

43 Aldo Di Virgilio, »L'offerta elettorale: La politica delle alleanze si istituzionalizza«, in: Roberto D’Alimonte / Stefano Bartolini (Hg.), Maggioritario finalmente? La transizione elettorale 1994 - 2001, Bologna 2002, S. 79-130, hier S. 80.

44 Ebenda, S. 126.

45 Stefan Köppl, Das politische System Italiens, aaO. (FN 29), S. 134.

46 Dazu wurden 100 Senatsabstimmungen vom 15.11.2006 bis zur Krisenabstimmung am 21. Februar analysiert. Als Grundlage dienen dabei die Abstimmungsinformationen, die auf der Homepage des Senates für jede einzelne Abstimmung abrufbar sind (www.senato.it). Insgesamt handelt es sich um 42 Regierungsvorlagen, während 55 von der Opposition stammen. Bei drei Vorlagen handelt es sich um Sonderabstimmungen, die die Rücktritte dreier Senatoren betreffen (Cossiga, Giaretta, Danieli). 
eine Konstellation, bei der den Senatoren auf Lebenszeit im Senat technisch betrachtet die Rolle als das Zünglein an der Waage zukommt. Die Regierung stützte sich auf eine knappe Mehrheit von gerade mal 158 zu 156 Senatoren $^{47}$. Die Niederlage am 21. Februar 2007 bei einer Abstimmung über den außenpolitischen Kurs war nur eine Frage der Zeit. Die Mehrheit der Regierung ist zu knapp, um mit der Breite der Koalition von elf Parteien ${ }^{48}$ verträglich zu sein. Während die beiden kommunistischen Senatoren Fernando Rossi und Franco Turigliatto nicht an der Abstimmung teilnahmen, trug das Verhalten der Senatoren auf Lebenszeit ebenfalls zur Niederlage der Regierung Prodi bei.

Bei den derzeitigen Senatoren auf Lebenszeit handelt es sich um die drei ehemaligen Staatspräsidenten Francesco Cossiga (Präsident von 1985 bis 1992; geboren 1928), Oscar Luigi Scalfaro (Präsident von 1992 bis 1999, geboren 1918) und Carlo Azeglio Ciampi (Präsident von 1999 bis 2006; geboren 1920). Außerdem amtieren der mehrfache Ministerpräsident und Symbolfigur der 1. Republik Giulio Andreotti (im Juni 1991 ernannt; geboren 1919), die Medizin-Nobelpreisträgerin Rita LeviMontalcini (seit August 2001; geboren 1909), der Unternehmer Emilio Colombo (seit Januar 2003; geboren 1920) und der Unternehmer Sergio Pininfarina (seit September 2005; geboren 1926). Sie gehören der gemischten Parlamentariergruppe (»misto«) an. Von den sechs anwesenden SaL stimmten drei für die Regierung (Ciampi, Levi-Montalcini, Colombo), einer dagegen (Cossiga) und zwei enthielten sich (Andreotti und Pininfarina). Enthaltungen erhöhen das Zustimmungsquorum und wirken wie eine Ablehnung. Dabei wurde vorher von einer Zustimmung von Andreotti und Pininfarina allgemein ausgegangen ${ }^{49}$. Gerüchteweise wollte sich Andreotti mit seinem Stimmungswechsel aber dafür rächen, dass Romano Prodi ihn zu Beginn der Legislaturperiode nicht als Senatspräsident durchgesetzt hat ${ }^{50}$.

Die detaillierte Auswertung zeigt zunächst, dass es im Untersuchungszeitraum neben der bekannten Abstimmung vom Februar 2007, noch zwei weitere gab, bei denen die Regierung keine Mehrheit der Senatoren hinter sich vereinen konnte - die

47 Stefan Köppl, »Die italienischen Parlamentswahlen 2006«, in: Zeitschrift für Parlamentsfragen, Nr. 4 (2006), S. 746-763, hier S. 759.

48 Im Senat setzt sich die Regierung Prodi zusammen aus den Linksdemokraten (62 Sitze), »La Margherita« (39 Sitze), »Rifondazione comunista« (27 Sitze), der gemeinsamen Liste von »Comunisti italiani Federazione dei Verdi« und »Consumatori uniti: Insieme con l’Unione« (11 Sitze), »Italia dei Valori« (4 Sitze), von »Populari-UDEUR «(3 Sitze) und jeweils einem Senator der »Lista Consumatori« und einem von Ulivo. Hinzukommen außerdem noch fünf Senatoren der Südtiroler Volkspartei aus der autonomen Region Süd-Tirol und einer aus dem Aosta-Tal. Daher sind es elf unterschiedliche politische Formationen, während der Abgeordnete aus dem Aosta-Tal eine Liste vertritt, die sich dort im Wesentlichen aus den nationalen Parteien zusammensetzt. Vgl. die Homepage des Senates unter www.senato.it.

49 Gianna Fregonara, »Lo schiaffo dei senatori a vita«, in: www.corriere.it, 22. Februar 2007.

50 Sebastiano Messina, »Andreotti e l'ombra della vendetta«, in: www.repubblica.it, 22. Februar 2007. 
Opposition allerdings auch nicht ${ }^{51}$. Daher haben diese beiden Ereignisse nicht das Gewicht, wie die spätere Niederlage der Regierung im Februar 2007. Grundsätzlich war der quantitative Einfluss der sieben Senatoren auf Lebenszeit eher gering, da sie meistens an den Abstimmungen nicht teilnahmen. Allerdings ergeben sich in diesem Punkt wesentliche Unterschiede zwischen den einzelnen SaL. Während die allgemeine Abwesenheitsquote bei circa 10\% lag, fehlten die Senatoren auf Lebenszeit im Durchschnitt bei circa 60\% der Abstimmungen. So hat Sergio Pininfarina nur an zwei von 100 Abstimmungen teilgenommen, während Emilio Colombo bei fast zwei Dritteln anwesend war. Der zweitaktivste ist Giulio Andreotti mit 56 von 100 Abstimmungen gewesen. Bei der 98-jährigen Rita Levi-Montalcini lag die Quote bei 45\%, während die ehemaligen Staatspräsidenten Cossiga und Ciampi nur 37mal abstimmten. Die Senatoren auf Lebenszeit enthielten sich mit 16,5\% zugleich öfter als ihre Kollegen mit 1,2\%. Da sie auch seltener als ihre Senatskollegen einer Vorlage zustimmten (37,7\% zu 54,1\%), während die Ablehnung in etwa gleich war (43,8\% zu 44,6\%), bevorzugten sie insgesamt häufiger den Status Quo. Die 100 untersuchten Abstimmungen waren in der Hauptsache Änderungsanträge (»emendamenti«), aber es hat auch fünf »votazione finale«, also Endabstimmungen über eine Vorlage gegeben. Diesen kommt eine größere Bedeutung als den einfachen Änderungsanträgen zu; allerdings nicht für die SaL. Gemäß dem Gesamtdurchschnitt waren sie nur $\mathrm{zu} 40 \%$ anwesend.

51 Dabei handelte es sich um zwei Änderungsanträge zum Haushaltsgesetz 1.130. Über den ersten wurde am 22. November 2006 abgestimmt. Bei 314 abstimmenden Senatoren lag das Quorum für die Annahme bei 158 Stimmen. Die Regierungskoalition stimmte mit 157 Senatoren dagegen, während es bei einer Enthaltung 156 Ja-Stimmen gab. Obwohl die Regierung keine absolute Mehrheit zustande brachte, scheiterte der Antrag dennoch, d.h. es war keine direkte Abstimmungsniederlage für die Regierung. Das gilt auch für den zweiten Fall vom 23. November 2006. Hier konnte die Koalition nur eine relative Mehrheit von 156 Stimmen aufbringen, während das Quorum bei 157 Stimmen lag. Befürwortet wurde dieser Antrag von 152 Senatoren, während sich vier enthielten. Homepage des Senates www.senato.it. 


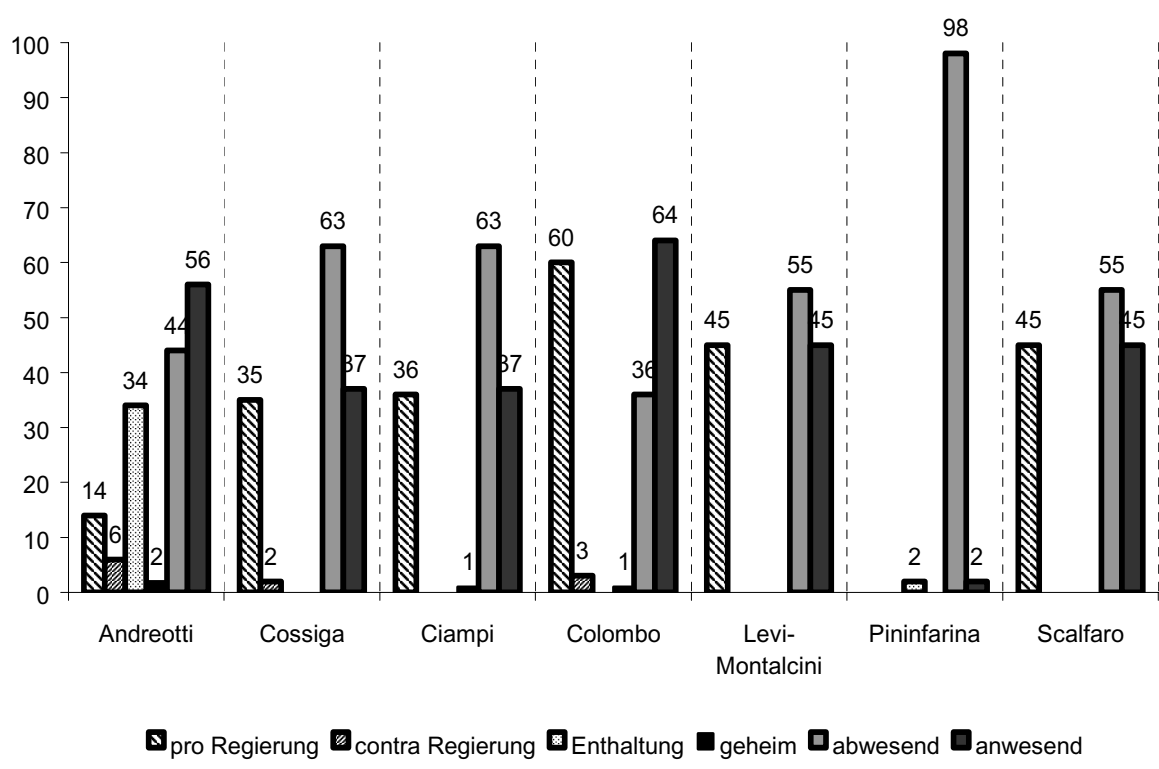

\section{Abbildung 2: Abstimmungsverhalten der Senatoren auf Lebenszeit im Untersuchungszeitraum im Verbältnis zur Regierung Prodi (Datenquelle: Homepage des Senates/Eigene Darstellung).}

Insgesamt votierten die $\mathrm{SaL}$ in $82 \%$ der Fälle (235-mal) für die Regierung Prodi, während die Opposition nur 11-mal (4\%) unterstützt wurde. Die Enthaltungsquote lag bei 13\% (36-mal) und geht auf Giulio Andreotti zurück (34-mal), der als einziger ein differenziertes Abstimmungsverhalten aufweist, wie die Abbildung 2 zeigt. Von Andreotti abgesehen, ist das Abstimmungsverhalten der anderen SaL sehr homogen. Oscar Luigi Scalfaro und Rita Levi-Montalcini haben ausschließlich für die Regierung abgestimmt. Die anderen drei haben lediglich ein oder zwei Mal eine geheime Wahl vollzogen. Dies betraf das Rücktrittsangebot ihres Kollegen Cossiga ${ }^{52}$. Ansonsten haben auch sie allein die Regierung unterstützt. Sergio Pininfarina hat nur zweimal sein Votum in der Aula abgegeben und sich beide Male inhaltlich enthalten. Es zeigt sich also, dass die Senatoren auf Lebenszeit größtenteils hinter der Regierung stehen und ihr ein zusätzliches Stimmenpolster verschaffen, während Andreotti der einzige ist, der ein differenziertes Abstimmungsverhalten aufweist. Demnach sind die Voten der Mehrheit der SaL berechenbar gewesen (nämlich bei

52 Der ehemalige Staatspräsident Francesco Cossiga hat am 27. November 2006 seinen Rücktritt als Senator auf Lebenszeit angeboten. Er begründete dies mit der Missachtung des Senates durch die Regierung, da diese einige seiner Anfragen, insbesondere zu dem umstrittenen Fall Abu Oma nicht beantwortet hätte. Ohne Autor, »Cossiga dà le dimssioni, il Senato le respinge«, in: www.corriere.it, 1. Februar 2007. 
Teilnahme zu Gunsten der Regierung), während Andreotti nach keinem erkennbaren Muster abstimmt.

Wie wären die Abstimmungen ohne die SaL ausgefallen? In 15 Fälle ist ihr Verhalten ohne Wirkung geblieben, da sie entweder nicht da waren oder sich ihre Voten gegenseitig aufhoben. Zweitens ergeben sich zehn Abstimmungen, bei denen ihr Abstimmungsverhalten zu Ungunsten der Regierung ausgefallen ist, da entweder durch direkte Gegenstimmen oder indirekt durch Enthaltungen die Opposition gestärkt wurde. Aber nur in dem Fall vom Februar führte dies zu einer Niederlage. In weiteren 45 Fällen hätte die Regierungsmehrheit ohne die Unterstützung der SaL eine Schwächung erfahren, die allerdings nicht zum Verlust der Mehrheit, sondern nur nahe an das Quorum heran geführt hätte.

$\mathrm{Zu}$ einem tatsächlichen Mehrheitsverlust der Regierung im Senat wäre es schließlich in 27 Fällen gekommen, wenn die SaL nicht mit ihren Stimmen die Regierung unterstützt hätten. Dabei handelt es ausschließlich sich um das Haushaltsgesetz 1132, über das am 22. und 23. November 2006 abgestimmt wurde. Die Senatoren auf Lebenszeit haben gezielt ihre Stimmen eingesetzt, um den Haushalt der Regierung Prodi durch zu bringen und somit für politische Stabilität gesorgt. Von 175 möglichen Voten bei den 25 zugehörigen Abstimmungen waren 104 zu Gunsten des Haushaltes der Regierung. Scalfaro und Levi-Montalcini haben dabei am massivsten mit 25 von 25 möglichen Voten die Regierung unterstützt. Es folgen Colombo mit 24 Stimmen, Cossiga mit 18 und Ciampi mit zehn Stimmen zu Gunsten des Haushaltes. Die Anwesenheitsquote der SaL bei den Abstimmungen über den Haushalt liegt mit 70,8\% dabei wesentlich über dem Gesamtdurchschnitt von etwa 40\%. Neben der Verabschiedung des Haushaltes gab es ansonsten nur eine Gelegenheit, bei der die Regierung nur Dank der SaL die Abstimmung überstanden hat ${ }^{53}$.

Angesichts dieser punktuellen Unterstützung des Haushaltes durch die SaL wird deutlich, dass ihr Abstimmungsverhalten davon geprägt ist, für politische Stabilität zu sorgen. Sie verhinderten, dass die erst neu gewählte Regierung Prodi nach nur wenigen Monaten im Amt scheiterte. Dass dies dennoch im Februar 2007 aufgrund der Enthaltung von Andreotti und Pininfarina geschah, zeigt die Janusköpfigkeit dieser Institution. Die Senatoren auf Lebenszeit sind keine homogene Gruppe, die ein identisches und vor allen Dingen kein berechenbares Abstimmungsverhalten an den Tag legt. Völlig vom demokratischen Wettbewerb um Unterstützung und damit auch um Legitimation abgekoppelt, vertreten die Senatoren auf Lebenszeit nur sich selbst.

\section{Die Zukunft der Senatoren auf Lebenszeit}

Die Institution der Senatoren auf Lebenszeit steht nach über zehn Jahren in der 2. Republik an einer Weggabelung. Die kommenden Ernennungen werden für ihren Charakter entscheidend sein. Werden auch in der 2. Republik hauptsächlich lang ge-

53 Nr. 49: Abstimmung über den Artikel 7 des Gesetzes Nr. 1184 vom 12.12.2006. Vgl. die Homepage des Senates unter www.senato.it. 
diente Parteipolitiker berufen, wie es bislang in der 1. Republik der Fall war, dann verschärft sich das Problem der Inkompatibilität von Herrschaftslogik der 2. Republik und der Institution der SaL. Denn dann wird, je nach Staatspräsident, irgendwann mit Silvio Berlusconi, Romano Prodi, Massimo D'Alema, Pier Ferdinando Casini oder Gianfranco Fini das heutige Spitzenpersonal auf Lebenszeit in den Senat einziehen. Die Folge wäre die schrittweise Vereinahmung der Institution der SaL durch die Parteipolitik. Die Parteien würden ihre ehemaligen Führungspersönlichkeiten nur zu gerne als zusätzliche Stimmenpolster in ihre Reihen eingliedern. Der scharfe Parteien(koalitionen)konflikt würde das tradierte Amtsverständnis überlagern. Durch die zunehmende Vereinnahmung durch die Parteipolitik werden die SaL zu unabwählbaren Parteivertretern, die eventuell ihre Rolle als Zünglein an der Waage vehement zu Gunsten ihrer Koalition einsetzen. Zumindest ist die Wandlung eines Berlusconi, sobald einmal ernannt, vom Saulus zum Paulus genauso unwahrscheinlich, wie sie es bei einem Andreotti gewesen ist. Ein positiver Effekt wäre allerdings, dass die Unberechenbarkeit im Stimmenverhalten der SaL reduziert werden würde, da dieses im Sinne ihrer Parteienkoalitionen erfolgen würde.

Vor diesem Hintergrund erscheinen nur zwei Strategien möglich, die sich abzeichnenden Reibungsverluste zu minimieren und zugleich die Institution der Senatoren auf Lebenszeit in ihrem ursprünglichen Sinne zu erhalten. So würde zum einen eine effektive Mehrheitsprämie für den Senat, die eben nicht auf regionaler Ebene eingerichtet ist, die Senatoren auf Lebenszeit wieder vom Zentrum an die Peripherie des parlamentarischen Prozesses im Senat bringen. Zweitens würde eine Ernennungspolitik, die ihren Schwerpunkt auf Persönlichkeiten von außerhalb der Politik legt, die parteipolitische Aufladung der Institution verhindern. Dieser Bruch mit sechs Jahrzehnten Ernennungstradition, ist aber angesichts der anhaltenden Macht der politischen Parteien eher unwahrscheinlich, so dass zumindest eine Veränderung ihres Selbstverständnisses mit kommenden Ernennungen wohl unvermeidlich sein wird. Wird diese nicht institutionell abgefedert (Mehrheitsprämie), werden die Senatoren auf Lebenszeit zunehmend zu unabwählbaren Parteipolitikern im Dienste ihrer Koalitionen.

\section{Schlussfolgerungen}

Für lediglich nominierte Parlamentarier verfügen die italienischen Senatoren auf Lebenszeit im internationalen Vergleich im Rahmen der Gleichberechtigung beider Kammern, als einzige über volle parlamentarische Kompetenzen. Lange Zeit war dies allerdings nicht von Bedeutung, da die SaL zur Zeit der 1. Republik aufgrund der dominierenden Herrschaftstechnik des »trasformismo "kaum mehr als eine symbolische Rolle spielten. Mit Beginn der eher mehrheitsdemokratisch geprägten 2. Republik unterliegen Bedeutung und Charakter der Institution der Senatoren auf Lebenszeit einem Wandel, der ihre Existenz massiv in Frage stellt, da sie im Ergebnis nicht mehr zur 2. Republik passt.

Den Senatoren auf Lebenszeit wird in einem Nullsummenspiel zwischen den rivalisierenden Parteienkoalitionen immer häufiger die Rolle als Zünglein an der 
Waage zufallen. Bei knappen Mehrheiten haben sie oder einzelne von ihnen, wie oben dargestellt, das Potenzial über den Bestand der Regierung zu entscheiden. Diese Machtfülle ist angesichts ihrer Bestellung und fehlenden Rückanbindung durch Wahlen oder über Parteien (die beiden abtrünnigen kommunistischen Senatoren wurden sofort ausgeschlossen) problematisch. Sie stellen in einem solchen Fall das letzte Stimmenreservoir für die rivalisierenden Parteienkoalitionen dar. Kam dieser Umstand bei der Einsetzung der ersten Regierung Berlusconi im Jahr 1994 bereits zum Tragen, so sind die SaL zu einer wichtigen Stütze der schwachen Regierung Prodi geworden. Dass die Regierung letztendlich über Andreotti und Pininfarina stolperte, betont die Uneinheitlich- und damit auch Unberechenbarkeit der Senatoren auf Lebenszeit. Die Ereignisse im Februar haben so die Schattenseite dieser Institution gezeigt, die solange in der 1. Republik verborgen blieb, wie es auf einzelne Stimmen nicht ankam. Doch nun kann ein politischer Nimmersatt wie Andreotti auf eigene Rechnung agieren.

Die Unberechenbarkeit wird sich allerdings mit der Zeit abschwächen, da die Ernennungstradition der Staatspräsidenten alt gediente Spitzenpolitiker auch der 2. Republik in den Senat auf Lebenszeit spülen wird. Damit wird diese Institution zunehmend entkernt und zum verlängerten Arm der Parteipolitik werden. Die wenigen Stimmen der SaL sind dann für die rivalisierenden Parteienkoalitionen zu kostbar, um sie noch in der vornehmen Sphäre der Überparteilichkeit zu belassen. Somit werden sich Bedeutung und Charakter der Institution im Übergang von der 1. zur 2. Republik diametral verändert haben, was nur noch einen Schluss übrig lässt. Entweder muss auch für den Senat eine effektive Mehrheitsprämie her, oder die Senatoren auf Lebenszeit sollten als das behandelt werden, was sie sind: Ein Relikt der 1. Republik.

\section{Zusammenfassung}

Der italienische Senat verfügt im Vergleich aufgrund der seltenen Kombination aus Direktwahl, unitarischem Staatsaufbau und parlamentarischer Vollkompetenz über ein intensives Legitimationsdilemma. Die zusätzliche Ernennung weniger Senatoren auf Lebenszeit ist angesichts der Pluralität der Verfahren anderer Zweiter Kammern weniger ungewöhnlich, als dass sie dabei über volle parlamentarische Kompetenzen verfügen. War dies in der 1. Republik noch zweitrangig, verändern sich in der 2. Republik Bedeutung und Charakter der Institution. Zeigte die Krise der Regierung Prodi noch ihre Unberechenbarkeit, droht eine Vereinnahmung durch die Parteipolitik, die einer Aushöhlung gleichkommt und diese Institution als ein Relikt der 1. Republik erkennen lässt.

\section{Summary}

Due to the direct election of $95 \%$ of its members and complete legislative powers as its main characteristics, the Italian Senate disposes of an intensive legitimation di- 
lemma within the unitarian Italian state. In the light of the diversity of other second chambers the additional nomination of a few Senators for life remains unproblematic. Only the specific conjunction of the nomination modus and the complete legislative powers leads to a unique gap in democratic legitimation. Negligible in the $1^{\text {st }}$ republic, the transition to the $2^{\text {nd }}$ Republic alters the significance and conception of the lifelong membership in the senate. While the crises of the Prodi government resulted from the unpredictability of this institution, the next nominations will expand the partisanship of the senators for life as part of the struggle of the two big party coalitions.

Eike-Christian Hornig, »Camera obscura - The Italian Senate and the new power of its lifetime members. 